\title{
Revealing pinning and solitonic transport of sliding charge-density-waves by coherent and nano-XRD
}

\author{
Vincent Jacques \\ CNRS / Laboratoire de Physique des Solides, Orsay, France; \\ vincent.jacques@u-psud.fr
}

The collective motion of electrons has always been a fascinating topic in condensed matter physics. In charge density wave (CDW) systems, transport measurements were the first to provide a clear signature of the collective motion of condensed electrons. A nonlinear conductivity is observed above a threshold current IT and is attributed to depinning of the CDW on impurities. An excess current then arises as well as a broad band noise and current oscillations. Although the electron density modulation involved in CDWs is very small, $\mathrm{x}$-ray diffraction provides information about the structure of the CDW as it is associated with a periodic lattice distortion. We will show here how state-of-the art X-ray diffraction techniques - coherent and nanoprobe XRD - can reveal the different steps of CDW deformations, from pinning to sliding, in systems of increasing dimensions.

Keywords: charge-density-wave, incommensurability, coherent x-ray diffraction, nanodiffraction 